\title{
Portable Space Mapping for Efficient Statistical Modeling of Passive Components
}

\author{
Lei Zhang, Member, IEEE, Peter H. Aaen, Senior Member, IEEE, and John Wood, Fellow, IEEE
}

\begin{abstract}
In this paper, a portable space mapping technique is presented for efficient statistical modeling of passive components. The proposed technique utilizes the cost-effective model composition of a statistical space mapping, while introducing the portable mapping concept for flexible model development for passive modeling. The portable mapping is a single-developmentmultiple-use versatile wrapper, such that after development it can be conveniently combined with any nominal model to form a set of statistical models of different speed and accuracy. This provides variety in model selection for different design needs. To further reduce modeling cost, i.e., the simulation time required for model data generation, a smart sampling technique is used to achieve better sampling fidelity with smaller sample size. The portable statistical mapping technique is demonstrated through modeling a transmission line and a spiral inductor.
\end{abstract}

Index Terms-Electromagnetic (EM), passive components, space mapping, statistical modeling

\section{INTRODUCTION}

$\mathrm{A}$ CCURATE statistical models are crucial in yield-driven design, where process parameter variations affect the performance of both on-wafer and packaged active and passive components [1]. As the operating frequency increases, the modeling of distributed effects of passive components becomes important [2], and it is necessary to examine the effects of process variations on these distributed passives for successful high-frequency circuit design. While passive nominal models can be obtained from full-wave electromagnetic (EM) simulations, which are computationally intensive, statistical analysis with EM simulations may be a time-prohibitive endeavor.

Research has been conducted on efficient statistical modeling methods, leading to a variety of techniques, such as equivalent circuit-based parameter extraction method [3], factor analysis [4], [5], and response surface methodology (RSM) [6], [7]. The equivalent circuit-based statistical model is developed through repetitive optimization-based parameter extractions. The link to component physical dimensions may

Manuscript received June 10, 2011, revised December 14, 2011.

L. Zhang and P. H. Aaen are with the RF Division, Freescale Semiconductor Inc., Tempe, AZ 85284 (e-mail: Lei.Zhang@freescale.com, Peter.Aaen@freescale.com).

J. Wood was with the RF Division, Freescale Semiconductor Inc., and he is now with Maxim Integrated Products, Inc., Sunnyvale, CA 94086 (e-mail: John.Wood@maxim-ic.com). be removed if the equivalent circuit model does not contain physics-based elements. Factor analysis searches for primary factors in process variations that contribute to the component behavior change. A response surface model can be built upon these critical factors by relating them to the component output responses of interest. This usually results in a mathematical model with no circuit representation.

Recently, space mapping-based techniques have emerged as a flexible alternative for statistical design and yield analysis of radio frequency (RF) and microwave circuits [8]-[12]. Initiated as an efficient optimization concept, space mapping has been successfully applied to the yield analysis of EMbased design [9], [10] and statistical modeling of nonlinear devices [11], [12], achieving accuracy at reduced simulation time. In [11] and [12], a novel formulation of space mapping, namely statistical space mapping, for statistical modeling of active devices, was presented, combining a nominal model and a mapping network to compose the statistical model. The nominal model represents the average behavior for a population of device samples and contains no statistical information. The manufacturing-process-initiated behavior difference between the samples is captured by the mapping network, which maps the model inputs into a new space where the nominal behavior is modified to include the statistical effects. Reliable statistical prediction of the model behavior has been reported.

In this paper, we demonstrate the application of space mapping for statistical passive component modeling. In the context of this paper, the "model" is described as a representation of a set of data, which are obtained through measurement or simulation of the passive component. A variety of passive models exists in the literature, such as equivalent circuit models from function fitting, outputs from EM simulations, or data from direct measurements. These different model formulations provide a list of choices for nominal models. In statistical space mapping, since the statistical behavior is captured entirely by the mapping network [12], which is a separate module from the nominal model, it is possible to apply the same statistical mapping to different nominal models. The portable mapping concept is introduced, and an approximate nominal model, such as an equivalent circuit model, is used for the fast statistical mapping extraction. The extracted mapping can be applied to a finer nominal model (2-D or 3-D EM simulation) to satisfy higher accuracy requirements. The accuracy and speed of the model is dependent on the nominal model and the statistical 
behavior is maintained by the mapping network.

This paper is organized as follows. In Section II, we reformulate the statistical space mapping to accommodate the passive modeling scenario. A smart sampling method, Latin Hypercube Sampling (LHS), is addressed in Section III for reliable statistical characterization. Through modeling passive components of a transmission line and a spiral inductor, Section IV demonstrates the portable statistical mapping technique as a reliable and cost-effective means to complement existing statistical modeling techniques.

\section{Portable Statistical MapPing for Passive Modeling}

\section{A. Formulation of Statistical Mapping Network}

In general, manufacturing process variations result in the changes of physical geometries and material properties, such as the length and width of a transmission line and the thickness and dielectric of the substrate. Statistical analysis is needed to model the behavior perturbation caused by these changes. Fast but approximate statistical models can be obtained in the form of equivalent circuit or physics-based models, and accurate statistical models can be obtained through EM simulations which could be computationally expensive. It is desired to have a statistical model that has both statistical accuracy and simulation speed.

We formulate a model whose response $y$ can be written as a function of the physical parameters $\boldsymbol{x}$ as $\boldsymbol{y}=\boldsymbol{g}(\boldsymbol{x})$ where $\boldsymbol{g}(\cdot)$ represents the behavior, $\boldsymbol{y}$, of the model with respect to the physical inputs $\boldsymbol{x}$. Inputs $\boldsymbol{x}$ may contain statistical variations, which directly deviates the nominal model behavior. We classify this category of parameters as explicit statistical variables. On the other hand, the statistical model response may also depend on another set of variables $\mathbf{z}$, which is not explicitly present in above model formulation. Such statistical parameter $\mathbf{z}$ is categorized as implicit statistical variables. In practice, the statistically explicit variables can be referred to geometrical parameters, such as the length and width of the transmission line, while the statistically implicit variables can be referred to material parameters such as the dielectric and loss tangent of the substrate. Implicit variables can be made explicit if the model formulation is changed to $\boldsymbol{y}=\boldsymbol{g}(\boldsymbol{x}, \boldsymbol{z})$. In this way, both $\boldsymbol{x}$ and $\mathbf{z}$ are explicit to the new model, which is by default statistically-capable. The proposed technique is to develop this new model formulation to incorporate the implicit statistical variables into the model's explicit parameters and enable a fully statistically-capable model.

In this paper, we propose to solve this modeling problem by extending the application of statistical space mapping [11], [12] from active modeling to passive modeling. To accommodate passive modeling needs, the statistical mapping is re-formulated such that the geometry variations are considered as explicit inputs to the mapping network, and the material variations are represented by the mapping coefficients, i.e., parameters of the mapping network. Thus, existing fast models, in terms of component geometries, can be used as the nominal model, whose behavior will be statistically perturbed by the mapping network. This modification complements the nominal model with statistical behavior due to both explicit (through inputs to mapping network) and implicit (through parameters of mapping network) variations.

Figure 1 illustrates the composition of the proposed statistical mapping model. Consider a statistical population of $N$ samples of a passive structure. Let $\boldsymbol{x}$ represent the geometry parameters of a passive component. Let $\phi^{k}, k=1,2, \ldots, N$, represent the mapping parameters for the $k^{\text {th }}$ sample. The statistical mapping network is formulated as

$$
\boldsymbol{x}_{\text {map }}^{k}=\boldsymbol{f}_{\text {map }}\left(\boldsymbol{x}, \boldsymbol{\phi}^{k}\right)
$$

where $\boldsymbol{x}_{\text {map }}$ denotes the new geometry space incorporating variations in both physical dimensions (explicit variables $x$ ) and material properties (implicit variables $\boldsymbol{z}$ that are explicitly represented by mapping parameters $\phi^{h}$ ). The mapping function $\boldsymbol{f}_{\text {map }}(\cdot)$ can be a simple linear function or more complicated nonlinear function depending on how big these variations are and how much they affect the component behavior. The nominal model outputs are modified by the statistically mapped geometry as

$$
\boldsymbol{y}^{k}=\boldsymbol{g}_{\text {nom }}\left(\boldsymbol{x}_{\text {map }}^{k}, \omega\right)
$$

where $\boldsymbol{g}_{\text {nom }}(\cdot)$ represents the nominal model, and $\boldsymbol{y}^{k}$ represents the response such as S-parameters at a given frequency $\omega$.

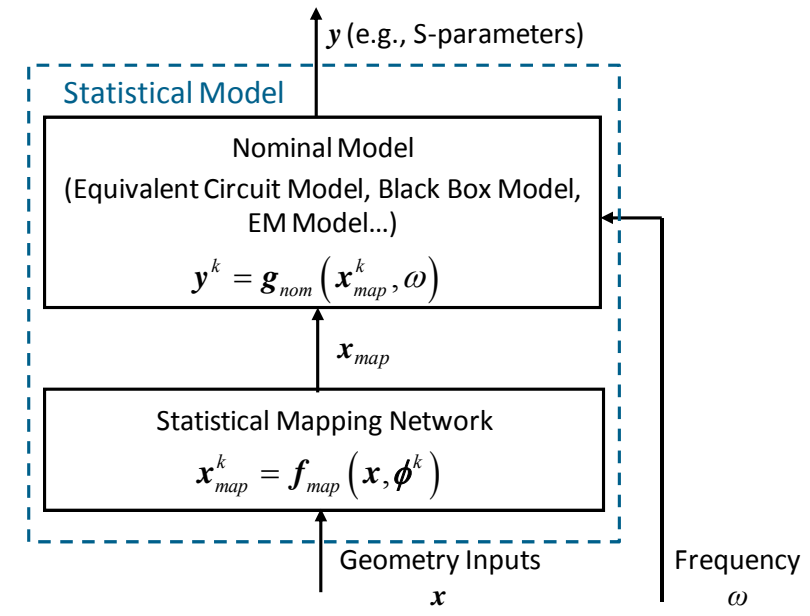

Fig. 1. Structure of the proposed statistical model composed of a nominal model and a statistical mapping network.

In this formulation, the behavior variation between component samples in a statistical population is captured only by the mapping network, given that the nominal model represents the average behavior of the population. The statistical difference (the behavior deviation from the nominal) can be independent of the nominal model. In other words, the statistical mapping network is "portable", that is, a mapping developed from a fast nominal model of "coarse accuracy" can be combined with a detailed EM simulation to produce 
"fine accuracy". This methodology of portable mapping can speed up statistical model development and reduce modeling cost when large numbers of EM simulations are required. It also allows use of statistically capable EM-based models to improve the yield of RF circuit.

\section{B. Statistical Mapping Extraction}

The core of this portable mapping technique is to find the statistical mapping parameters. This is achieved through an optimization-based parameter extraction procedure as in [12], where the mapping parameters are adjusted to minimize the difference between model outputs and component data for each sample in the statistical population. A fast nominal model is preferable for this step to avoid heavy computational cost by detailed models. Figure 2 shows the optimization diagram.

Define $y_{d}^{k}$ as the component data for the $k^{\text {th }}$ statistical sample. The optimization objective is to minimize the difference between data and the model response

$$
\min _{\phi^{k}}\left(\left\|\boldsymbol{y}_{d}^{k}-\boldsymbol{g}_{\text {nom }}\left(\boldsymbol{f}_{\text {map }}\left(\boldsymbol{x}, \boldsymbol{\phi}^{k}\right), \omega\right)\right\|^{2}\right)
$$

by finding $\phi^{k}, k=1,2, \ldots, N$, for each sample in the population. After all $\phi^{k}$ s s are found, their means $(\mu)$, standard deviations $(\sigma)$, and correlations $(\rho)$ are computed and become the statistical equivalents to the material variations. These statistical quantities of mapping coefficients, together with the geometrical inputs, form the statistical mapping network, which can then be combined with the nominal model to build the overall statistical model.

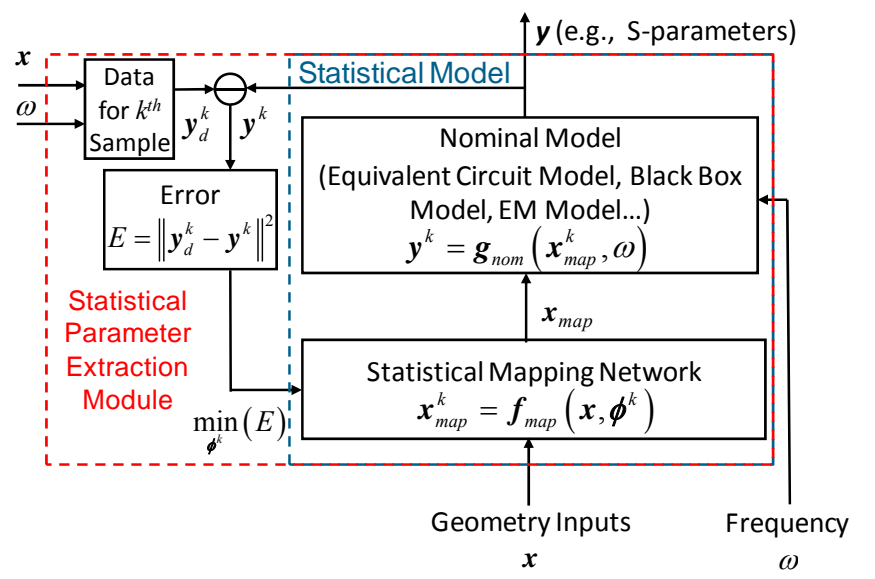

Fig. 2. Diagram for developing statistical mapping network through optimization-based parameter extractions.

\section{Use of the Statistical Model}

The component we modeled may have different forms of nominal models. Once the statistical mapping is developed, it can be applied to other forms of nominal models to compose a statistical model ready for design use. Depending on the design requirement, the nominal model can be at the same level of speed and accuracy as the one used in statistical mapping extraction, or a more accurate model at the cost of simulation speed. The mathematical mapping formulation is simulator independent and convenient to implement. It fully represents the statistical distributions in both physical geometries and material properties, and can effectively produce statistical estimations for RF passive components.

\section{Discussion}

Let's consider a linear passive modeling problem where statistical perturbation to the component structure is relatively small to the nominal structure. For a random structure under such assumption, the output response $y$ with respect to a given physical input $\boldsymbol{x}$ can be represented by $\boldsymbol{g}(\boldsymbol{x})$. Define the statistical difference between this random structure and the nominal structure as $\Delta \boldsymbol{x}=\boldsymbol{x}-\boldsymbol{x}_{\text {nom }}$, where $\boldsymbol{x}_{\text {nom }}$ represents the physical input of the nominal structure, and the output response can be further expanded by using Taylor series as:

$$
\begin{aligned}
\boldsymbol{y} & =\boldsymbol{g}\left(\boldsymbol{x}_{\text {nom }}+\Delta \boldsymbol{X}\right) \\
& =\boldsymbol{g}\left(\boldsymbol{x}_{\text {nom }}\right)+\left.\frac{\partial \boldsymbol{g}}{\partial \boldsymbol{x}}\right|_{\boldsymbol{x}=\boldsymbol{x}_{\text {nom }}} \cdot \Delta \boldsymbol{X}+\left.\frac{\partial^{2} \boldsymbol{g}}{\partial \boldsymbol{x}^{2}}\right|_{\boldsymbol{x}=\boldsymbol{x}_{\text {nom }}} \cdot(\Delta \boldsymbol{x})^{2}+\cdots
\end{aligned}
$$

With the perturbation being small, a first-degree linear polynomial can be sufficient to approximate the behavior variation between a random sample and the nominal, by removing the 2 nd and higher order terms in (4),

$$
\boldsymbol{y}-\boldsymbol{y}_{\text {nom }}=\boldsymbol{y}-\boldsymbol{g}\left(\boldsymbol{x}_{\text {nom }}\right)=\left.\frac{\partial \boldsymbol{g}}{\partial \boldsymbol{x}}\right|_{\boldsymbol{x}=\boldsymbol{x}_{\text {nom }}} \cdot \Delta \boldsymbol{X} .
$$

In (5), $\left.\frac{\partial \boldsymbol{g}}{\partial \boldsymbol{x}}\right|_{\boldsymbol{x}=\boldsymbol{x}_{\text {nom }}}$ is only dependent on the nominal structure and the model representation function $\boldsymbol{g}$. It is seen that the statistical perturbation from the input to the nominal behavior resides only in $\boldsymbol{\Delta} \boldsymbol{X}$ and independent of the nominal model. In this sense, the statistical mapping is not only portable to different nominal models for this one physical structure of the passive component being modeled, but it can also be portable to this passive component with different physical dimensions. For example, the statistical wrapper could be extracted on an inductor with one set of geometrical inputs, and the wrapper could be applied to a different set of geometrical inputs, as will be shown later in Section IV. When the behavior deviation from the nominal is large and higher-degree polynomials are necessary, the mapping is portable to different nominal models of one structure but not to different structures.

If implicit variables are present and their statistical variations are also small, a multivariable Taylor series can be applied to the model response at given explicit input $\boldsymbol{x}$ and implicit input $\mathbf{z}$. As in (1), the implicit variables are explicitly represented by mapping parameters $\phi$, whose extracted values and statistical information are fictitious representations of the implicit inputs. In this case, output response of a random structure can be defined as $\boldsymbol{y}=\boldsymbol{g}\left(\boldsymbol{f}_{\text {map }}(\boldsymbol{x}, \phi)\right)$. If the small variations in the implicit inputs can be propagated into the mapping coefficients $\phi$, (5) can be expanded to accommodate the implicit inputs as 


$$
\begin{aligned}
\boldsymbol{y}-\boldsymbol{y}_{\text {nom }} & =\boldsymbol{y}-\boldsymbol{g}\left(\boldsymbol{f}_{\text {map }}\left(\boldsymbol{x}_{\text {nom }}, \boldsymbol{\phi}_{\text {nom }}\right)\right) \\
& =\left.\frac{\partial \boldsymbol{g}}{\partial \boldsymbol{f}_{\text {map }}} \cdot \frac{\partial \boldsymbol{f}_{\text {map }}}{\partial \boldsymbol{x}}\right|_{\boldsymbol{x}=\boldsymbol{x}_{\text {nom }}} \cdot \Delta \boldsymbol{X}+\left.\frac{\partial \boldsymbol{g}}{\partial \boldsymbol{f}_{\text {map }}} \cdot \frac{\partial \boldsymbol{f}_{\text {map }}}{\partial \boldsymbol{\phi}}\right|_{\boldsymbol{\phi}=\boldsymbol{\phi}_{\text {oum }}} \cdot \Delta \boldsymbol{\phi}
\end{aligned}
$$

where $\phi_{n o m}$ is the nominal representation of the implicit inputs, and $\Delta \phi=\phi-\phi_{n o m}$ represents the implicit random deviation from nominal. In (6), the first order derivatives $\frac{\partial \boldsymbol{g}}{\partial \boldsymbol{f}_{\text {map }}}, \frac{\partial \boldsymbol{f}_{\text {map }}}{\partial \boldsymbol{x}}$, and $\frac{\partial \boldsymbol{f}_{\text {map }}}{\partial \phi}$ are evaluated at the nominal input values. The statistical behavior deviation from the nominal only relies on $\Delta \boldsymbol{X}$ and $\Delta \boldsymbol{\phi}$, and independent of nominal model. Thus the portability of the mapping to different nominal models and different physical dimensions still hold.

We tend to formulate the mapping using linear equations to simplify the model computation. This is usually applicable if nominal models are properly chosen, for example, passive structures whose models have reasonably good accuracy. This results in small behavior variations between random samples, which can be well captured by a simple linear network, as we will illustrate in Section IV. Nonlinear mapping may be needed if the nominal model is inappropriately chosen and does not accurately exhibit the average behavior, or the statistical variations are large enough causing random samples to differ greatly from the nominal behavior.

Note that in Fig. 1, the frequency parameter was considered as the input to the nominal model and not an input to the mapping network. This structure can be modified to accommodate frequency-sensitivity mapping [8] by adding the frequency as an extra input to the mapping network, which then produces a frequency-dependent geometrical space. This can expand a nominal model usage from its developing frequency to different frequency regions, which is useful for model enhancement, though this may impact the portability of the statistical wrapper.

\section{LATIN HyPERCUBE SMART SAMPLING}

Statistical modeling often involves sampling the input space to form a random population through selection of limited number of samples. This population becomes a statistical representation of the manufacturing process induced variations, and the samples in the population are used to generate data for statistical model development. A straightforward sampling technique is the simple random sampling (SRS) [13], which randomly picks samples from some prescribed probability distributions of the input space. This method usually requires hundreds of samples to achieve a good coverage of multidimensional input space for accurate estimation of statistical variations. In reality, data generation by hundreds of measurements or EM simulations is very timeconsuming and often prohibitive.

In computer modeling, smart sampling techniques [14] have been developed as efficient alternatives to simple random sampling. Such techniques provide a systematic means in choosing important samples to build a population with improved coverage of the input space. In this way, a smaller population from smart sampling may produce the same or better statistical representation as an intensively sampled population from simple random sampling does. In this paper, we adopt a smart sampling technique, the Latin Hypercube Sampling [14], [15], to statistical passive modeling. In addition to have improved efficiency over simple random sampling as other stratified smart sampling methods, Latin hypercube sampling technique also ensures a full coverage of the range of each variable by maximally stratifying each marginal distribution, and appears to be a good method to use for selecting values of input variables [14]. It is efficient in sampling input variables for reliable statistical estimation and cost-effective data generation.

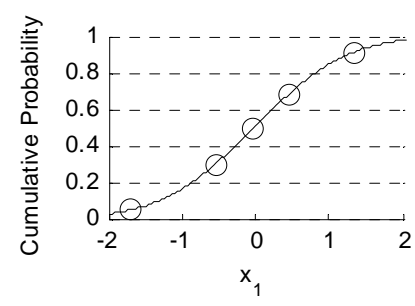

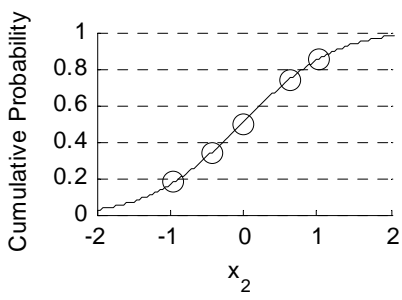

(a)

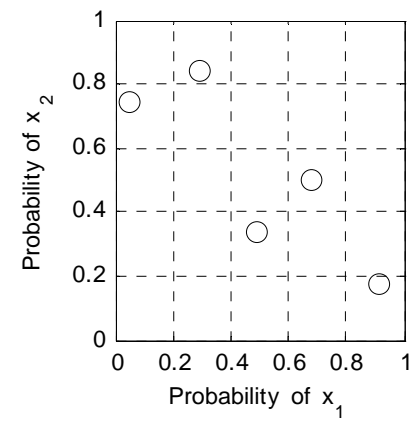

(b)
Fig. 3. Example of LHS: (a) Random stratified sampling of variables $x_{1}$ and $x_{2}$ at 5 intervals and (b) random pairing of sampled $x_{1}$ and $x_{2}$ forming a Latin hypercube.

Consider an $M$-dimensional input space $\boldsymbol{x}$ of the passive component. Latin hypercube sampling involves sampling $N$ values from the prescribed distribution of each of the $M$ input variables. The cumulative distribution for each variable is divided into $N$ equiprobable intervals. A value $x_{l}^{k}$, $l=1,2, \ldots, M$ and $k=1,2, \ldots, N$ is selected randomly from each interval for each variable. The $N$ values obtained for $l^{\text {th }}$ variable $\left\{x_{l}^{1}, x_{l}^{2}, \ldots, x_{l}^{N}\right\}$ are then paired with the other variables based on the correlation matrix of the input variables [16] to form $N$ samples of input vectors $\left\{x_{1}, x_{2}, \ldots, x_{M}\right\}^{k}$.

Figure 3 illustrates this sampling procedure with an example of $M=2$ and $N=5$, where both inputs have Gaussian distribution with mean values $\boldsymbol{\mu}=\left[\begin{array}{ll}0 & 0\end{array}\right]$, standard devations $\boldsymbol{\sigma}=\left[\begin{array}{ll}1 & 1\end{array}\right]$, and correlation matrix $\boldsymbol{\rho}=\left[\begin{array}{cc}1 & 0.5 \\ 0.5 & 1\end{array}\right]$. 


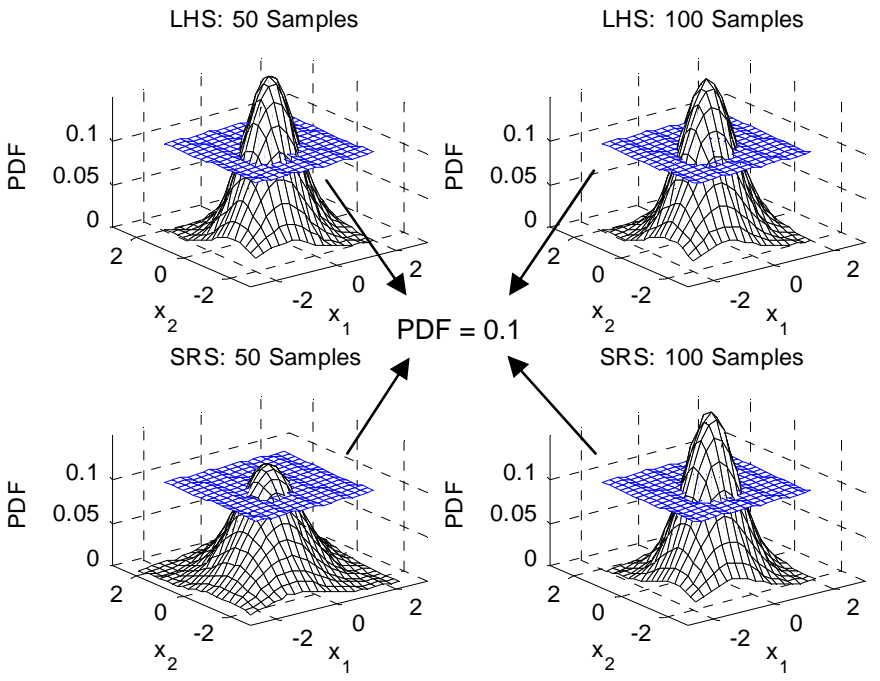

(a)

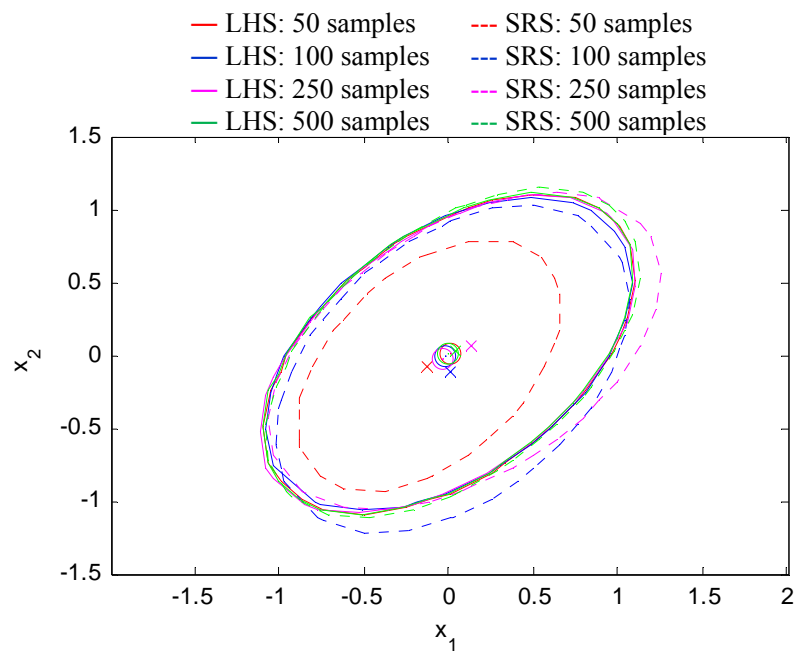

(b)

Fig. 4. Comparison between LHS and SRS using a two-input sampling example with zero mean values, unit standard deviations, and correlation coefficient of 0.5 between the two inputs $x_{1}$ and $x_{2}$. (a) Sample surface plots of probability density function of $\boldsymbol{x}$ with 50 and 100 samples from LHS and SRS. (b) Contour plots at PDF $=0.1$ ( - for LHS and --- for SRS) and at maximum PDF ( $x$ for LHS and o for SRS) for 4 different population sizes. LHS contours of all 4 populations align well with each other, showing that only 50 LHS samples can provide good coverage of the overall input space.

To demonstrate the advantage of Latin hypercube sampling over simple random sampling, sampling experiments are performed for the above 2-input example shown in Fig. 3. Four populations with different sample sizes of 50, 100, 200, and 500 are generated using each sampling method. Figure 4(a) shows the surface plot of the probability density function (PDF) for the 50 and 100 population sampled using simple random sampling, as compared to that from Latin hypercube sampling in Fig. 4(b). It is observed that in Fig. 4(a), the peak of the SRS PDF changes for the two sample sizes we chose to plot, while the peaks of the LHS PDF stay close. We also plot the contours at $\mathrm{PDF}=0.1$ for all populations. As depicted in Fig. 4(b), the probability density contours for $50,100,250$, and 500 samples from Latin hypercube sampling almost align with each other, while the probability density contours from the simple random sampling exhibit difference. This shows that the Latin hypercube sampling provides more reliable coverage of the input space with much smaller sample size compared to the simple random sampling.

\section{EXAMPLES}

\section{A. Portable Mapping for Transmission Line Modeling}

To demonstrate the proposed statistical modeling approach, we select a pedagogical example in which we have the statistical model and try to duplicate it using our technique. A microstrip transmission line is studied where geometric and material parameters, along with their nominal values, are listed in Table I. The "MLIN" circuit component in Agilent EDA software, Advanced Design System $\left(A D S^{\mathrm{TM}}\right)$, is considered as the component to be modeled. MLIN is a frequency-domain analytical model which uses closed form formulas to calculate the transmission line parameters such as the static impedance, the effective dielectric constant, and the attenuation factor, thus the scattering parameters of the line. The physical dimensions to be varied for MLIN are the length $(l)$ and width $(w)$ of the microstrip, the substrate height $(h)$, dielectric constant $\left(\varepsilon_{r}\right)$, and loss tangent $(\tan \xi)$. The Gaussian distribution is presumed for all these 5 parameters, with $\pm 5 \%$ standard deviations around their nominal values.

TABLE I

PhySiCAL PARAMETERS OF THE MiCROSTRIP STRUCTURE

\begin{tabular}{|c|c|c|c|}
\hline \hline \multicolumn{2}{|c|}{ Parameters } & $\begin{array}{c}\text { Nominal Value } \\
(\boldsymbol{\mu})\end{array}$ & $\begin{array}{c}\text { Standard Deviation } \\
(\boldsymbol{\sigma})\end{array}$ \\
\hline \multirow{2}{*}{ Microstrip } & Length $(l)$ & $100 \mathrm{mil}$ & $5 \mathrm{mil}$ \\
\cline { 2 - 4 } & Width $(w)$ & $25 \mathrm{mil}$ & $1.25 \mathrm{mil}$ \\
\hline \multirow{3}{*}{ Substrate } & Height $(h)$ & $10 \mathrm{mil}$ & $0.5 \mathrm{mil}$ \\
\cline { 2 - 4 } & Dielectric Constant $\left(\varepsilon_{r}\right)$ & 9.6 & 0.48 \\
\cline { 2 - 4 } & Loss Tangent $(\tan \xi)$ & 0.0002 & $1 \mathrm{e}-5$ \\
\hline \hline
\end{tabular}

A statistical model is built following the formulation in Section II. $A$. The MLIN circuit model with nominal substrate parameters is deliberately picked as the nominal model, with the assumption that only geometrical parameters, $l$ and $w$, are explicit inputs. The statistically perturbed substrate parameters $h, \varepsilon_{r}$, and $\tan \xi$ are considered as implicit inputs. To capture the behavior deviation due to variations in the substrate parameters, a statistical mapping network is developed whose mapping parameters become the explicit representation of the implicit substrate parameters. We formulate the statistical mapping as a simple linear function of (1)

$$
\begin{aligned}
& l_{\text {map }}^{k}=a_{1}^{k} \cdot l+a_{2}^{k} \cdot w \\
& w_{\text {map }}^{k}=b_{1}^{k} \cdot l+b_{2}^{k} \cdot w
\end{aligned}
$$

where $\boldsymbol{\phi}^{k}=\left\{\boldsymbol{a}^{k}, \boldsymbol{b}^{k}\right\} \quad(k=1,2, \ldots, N)$ contains the mapping parameters for the $k^{\text {th }}$ sample. Note that $h, \varepsilon_{r}$, and $\tan \xi$ are not present in (7) but indirectly represented by $\boldsymbol{\phi}^{k}$, to be found for each sample through statistical mapping extraction. After 
extraction, the means, standard deviations, and correlations of extracted $\phi^{k}$ s are used to describe the substrate variations.

For statistical model development, a population of $N=50$ samples is generated using the Latin hypercube sampling by varying the physical parameters in Table I. As described in Section III, we expect this population size to efficiently sample the overall input space and small enough as in practical experiment. This population is for model development and is called "training population". Small-signal S-parameter simulations are performed at each sample in the training population, whose responses are called "training data" and used for statistical mapping extraction following the optimization procedure of Fig. 2. The means and standard deviations of the extracted $\boldsymbol{a}$ 's and $\boldsymbol{b}$ 's are shown in Table II. The correlation matrix of the extracted parameters is

$a_{1}$
$a_{1}$
$a_{2}$
$b_{1}$
$b_{2}$$\left[\begin{array}{cccc}1.0 & -0.1012 & 0.1350 & 0.5069 \\ -0.1012 & 1.0 & -0.5316 & -0.0843 \\ 0.1350 & -0.5316 & 1.0 & 0.0610 \\ 0.5069 & -0.0843 & 0.0610 & 1.0\end{array}\right]$

TABLE II

EXTRACTED STATISTICS OF THE MAPPING PARAMETERS FOR THE TRANSMISSION LINE EXAMPLE

\begin{tabular}{|c|c|c|}
\hline \hline Mapping Parameters & Mean Values $(\boldsymbol{\mu})$ & Standard Deviation $(\boldsymbol{\sigma})$ \\
\hline$a_{1}$ & 0.9978 & 0.0685 \\
\hline$a_{2}$ & $8.6988 \mathrm{e}-15$ & $6.7462 \mathrm{e}-16$ \\
\hline$b_{1}$ & $3.0122 \mathrm{e}-10$ & $3.3938 \mathrm{e}-11$ \\
\hline$b_{2}$ & 1.0176 & 0.1950 \\
\hline \hline
\end{tabular}
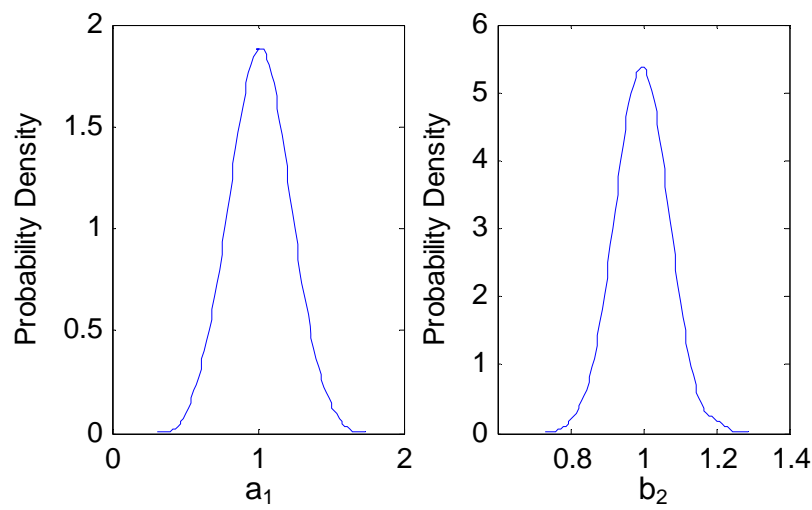

Fig. 5. Probability density plot of the extracted mapping parameters. Gaussian distribution is observed for the transmission line example.

As observed from (8) and Table II, $a_{2}$ and $b_{1}$ have very small values compared to $a_{1}$ and $b_{2}$, and their correlations with $a_{1}$ and $b_{2}$ are also small. This indicates that $a_{1}$ and $b_{2}$ are two dominating factors of the statistical mapping, while $a_{2}$ and $b_{1}$ are less important thus can be neglected or removed to simplify the mapping network. This is as expected, because the length and width of the transmission line are uncorrelated. Fig. 5 shows the probability density plot of the extracted $a_{1}$ and $b_{2}$. Notice that in this example, the mapping parameters follow Gaussian distribution. If the distributions of the extracted mapping parameters are irregular, a normal transformation may be needed to convert the extracted distribution to Gaussian [12].

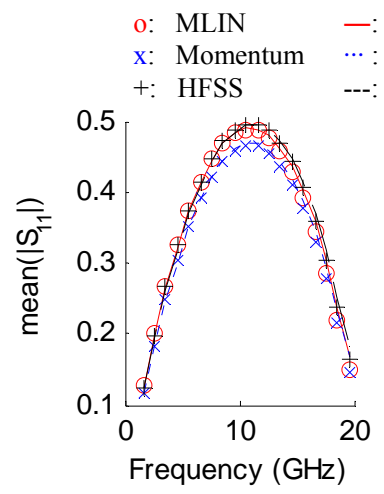

—: Mapped MLIN Model

$\cdots$ : Mapped Momentum Model

--: Mapped HFSS Model
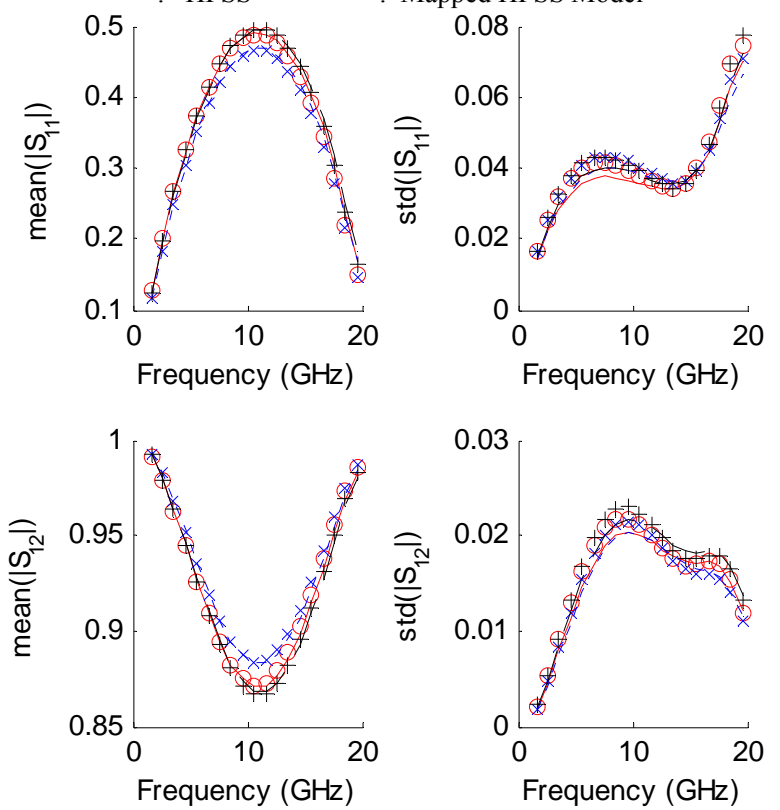

(a)
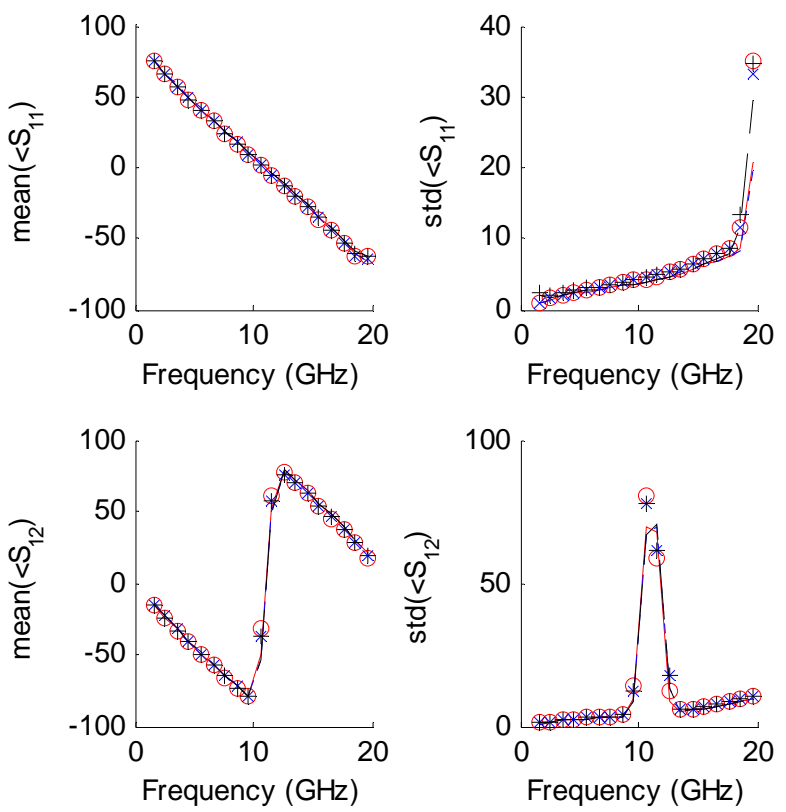

(b)

Fig. 6. Means and standard deviations of (a) magnitude of $\mathrm{S}_{11}$ and $\mathrm{S}_{12}$, and (b) phase components of $S_{11}$ and $S_{12}$ for the microstrip example. Comparison is between the test population and the statistical mapped model. The same mapping developed from MLIN nominal model is applied to two other nominal models using Momentum and HFSS simulation data. The portable statistical mapping successfully captured the statistical behavior for all three nominal models.

To test the statistical mapping, we generated another population of 200 samples using Latin hypercube sampling with the same distribution in Table I. This population is used 
to verify the statistical model and thus called the "test population", and the corresponding 200 sets of S-parameter data are called "test data". Using the statistical means and variances in Table II and (8), which are extracted from the 50 training samples, another 200 sets of mapping parameters are generated. These mapping parameters, together with nominal model, compose the statistical model for test. To demonstrate the portability of the mapping network, we apply the mapping to three different nominal models: the ADS MLIN circuit model used for model development, and two other models with fine EM accuracy obtained from two EM simulators: ADS Momentum and HFSS ${ }^{\mathrm{TM}}$. All three nominal models have the same physical parameters with nominal values in Table I.

For statistical verification, the three models represented by the MLIN equivalent circuit, the output data from Momentum simulation, and the output data from HFSS simulation are evaluated using the same physical parameters in the test population. Their small-signal S-parameters are compared with those computed from the statistical models using different nominal models. As shown in Fig. 6, the portable mapping is able to statistically perturb the nominal model to reproduce the statistical means and standard deviations of the small-signal responses independent of the nominal model. The correlation between the S-parameters is also successfully reassembled using the portable mapping as demonstrated in Table III.

TABLE III

SAMPLES OF CORRELATION COEFFICIENTS FROM THE S-PARAMETERS OF THE TRANSMISSION LINE EXAMPLE

\begin{tabular}{|c|c|c|c|}
\hline \hline & \multicolumn{3}{|c|}{ Correlation Coefficients } \\
\cline { 2 - 4 } & $\left(\mathrm{RS}_{11}, \mathrm{IS}_{11}\right)$ & $\left(\mathrm{RS}_{11}, \mathrm{RS}_{12}\right)$ & $\left(\mathrm{RS}_{11}, \mathrm{IS}_{12}\right)$ \\
\hline MLIN & -0.0420 & 0.1619 & 0.9370 \\
\hline Mapped MLIN Model & -0.0508 & 0.2015 & 0.9396 \\
\hline Momentum & -0.0474 & 0.1791 & 0.9362 \\
\hline Mapped Momentum Model & -0.0570 & 0.2184 & 0.9380 \\
\hline HFSS & -0.0554 & 0.1864 & 0.9356 \\
\hline Mapped HFSS Model & -0.0646 & 0.2238 & 0.9374 \\
\hline \hline
\end{tabular}

The portability of the mapping network is further tested by wrapping it around other nominal structures. Two new transmission lines with different nominal parameters from the original structure are used, with lengths of $80 \mathrm{mil}$ and $50 \mathrm{mil}$, width of 20 mil and 30 mil, substrate height of 8 mil and 5 mil, dielectric constant of 10.8 , and loss tangent of $1.5 \mathrm{e}-4$. These two lines have characteristic impedance $Z_{o}$ of $26 \Omega$ and $17 \Omega$, respectively, compared to the $30 \Omega$ line in Table II. Two populations of 100 samples are generated for both of these new transmission lines using the same distribution as the original line, and the same mapping of Table II and (8) is applied to the corresponding MLIN nominal models. As illustrated in Fig. 7, the statistical behaviors are accurately captured for both transmission lines, by the mapping extracted from a different (original) line structure.

Another important benefit of the portable mapping technique is the cost-effective model development using a fast nominal model. To illustrate this, we compared modeling cost by recording statistical parameter extraction time for a population of 100 samples, using both a fast circuit model and a slow EM simulation as nominal model. The test was performed with circuit and Momentum simulations in $A D S^{\mathrm{TM}}$ on a PC with Intel ${ }^{\circledR} \mathrm{Core}^{\mathrm{TM}} 2$ Duo CPU at $3 \mathrm{GHz}$. The overall modeling time was 42.70 seconds using the fast nominal model, compared to 18.89 minutes using the slow nominal model. Yet the statistical mapping extracted from the fast nominal model can provide the required statistical accuracy in a fraction of time. As EM complexity increases, the speedup in modeling by the portable mapping can become dramatic.

$\begin{array}{lll}\text { o: } & \text { New Microstrip Structure \#1 } & \text { - : Mapped Model for Microstrip \#1 } \\ \text { x: } & \text { New Microstrip Structure \#2 } & \text {--- : Mapped model for Microstrip \#2 }\end{array}$
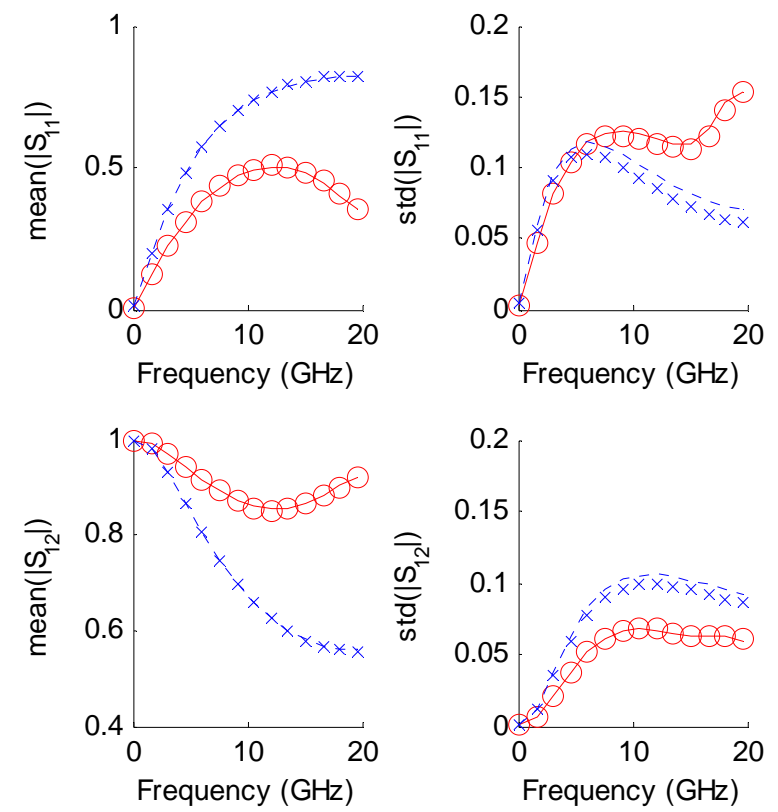

(a)
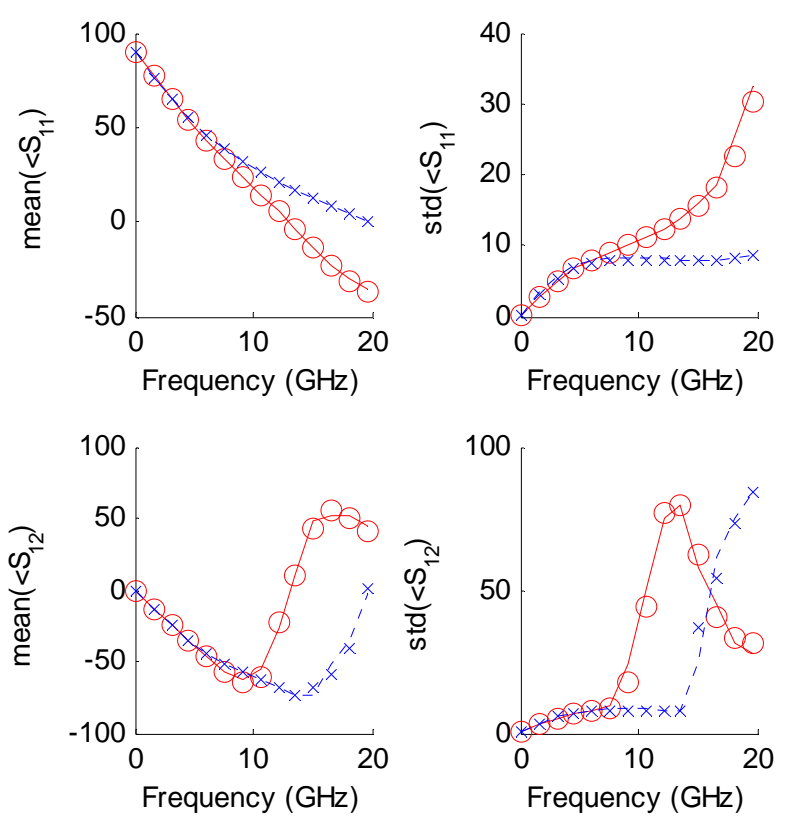

(b)

Fig. 7. Means and standard deviations of (a) magnitude of $\mathrm{S}_{11}$ and $\mathrm{S}_{12}$, and (b) phase components of $S_{11}$ and $S_{12}$ for two different microstrip structures. Comparison is between the test population and the statistical mapped model. The portable statistical mapping developed from the original microtrip successfully captures the statistical behavior for both new structures. 


\section{B. Statistical Modeling of a Spiral Inductor}

Spiral inductors are popularly used in RF circuit as matching elements. For accurate design, it is necessary to have them properly characterized to include manufacturing process variation effects. In this example, the portable statistical mapping is used for statistical modeling of a spiral inductor, whose structure is shown in Fig. 8(a) drawn on the technology specific substrate and with its parameters defined in Table IV. We consider the line width and longitudinal inside diameter as the explicit statistical inputs, while the spacing and transverse inside diameter are the implicit statistical inputs. The nominal model used for statistical mapping development is an equivalent circuit based model as shown in Fig. 8(b), where the values of resistors, inductors and capacitors are functions in terms of the physical parameters extracted from EM data.

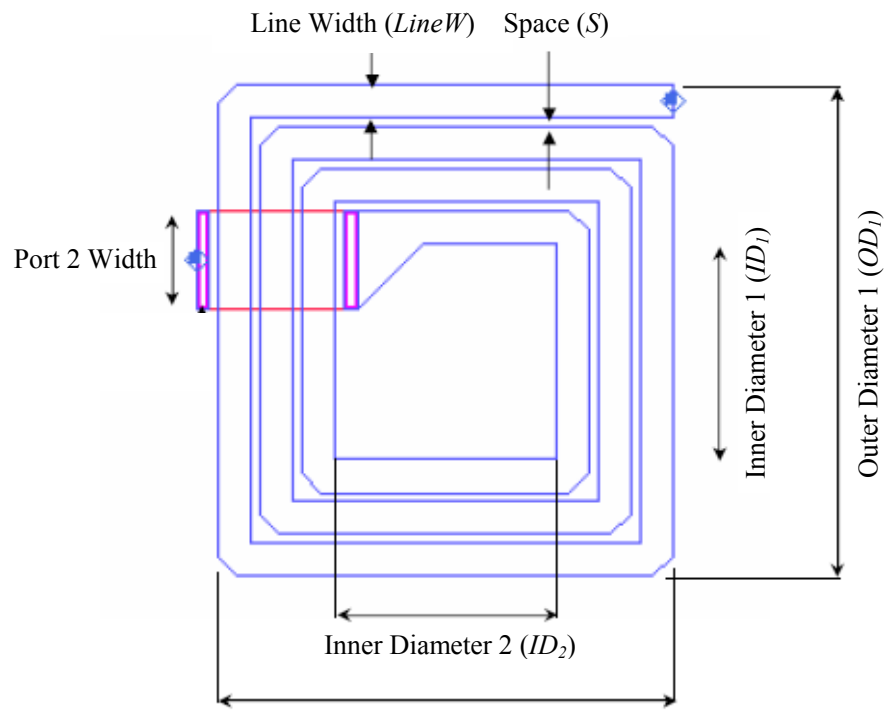

Outer Diameter $2\left(\mathrm{OD}_{2}\right)$

(a)

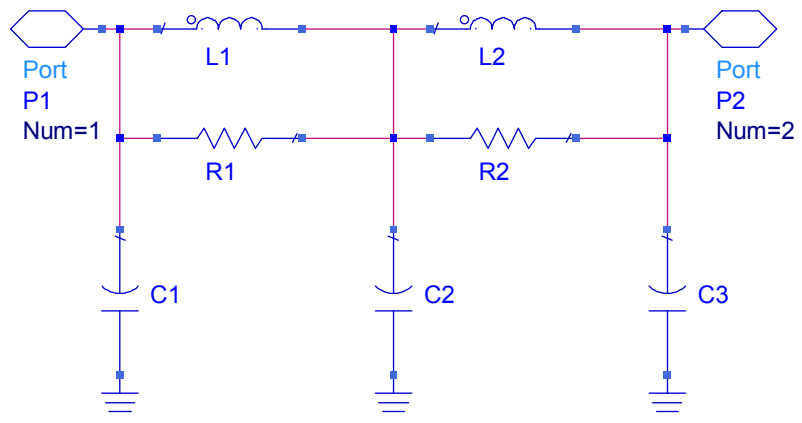

(b)

Fig. 8. (a) Structural definition of a spiral inductor model, and (b) equivalent circuit representation of the nominal model for the inductor.

TABLE IV

PHYSICAL PARAMETERS OF THE SPIRAL INDUCTOR

\begin{tabular}{|c|c|c|}
\hline \hline Parameters & $\begin{array}{c}\text { Nominal Value } \\
(\boldsymbol{\mu})\end{array}$ & $\begin{array}{c}\text { Standard Deviation } \\
(\boldsymbol{\sigma})\end{array}$ \\
\hline Line Width $($ LineW $)$ & $15 \mu \mathrm{m}$ & $1.5 \mu \mathrm{m}$ \\
\hline Line Spacing $(S)$ & $6 \mu \mathrm{m}$ & $0.6 \mu \mathrm{m}$ \\
\hline Longitudinal Inside Diameter $\left(I D_{l}\right)$ & $100 \mu \mathrm{m}$ & $10 \mu \mathrm{m}$ \\
\hline Transverse Inside Diameter $\left(I D_{2}\right)$ & $100 \mu \mathrm{m}$ & $10 \mu \mathrm{m}$ \\
\hline \hline
\end{tabular}

To study the statistical behavior of the spiral inductor, we perturbed the physical parameters with the statistical specifications in Table IV, and used Latin hypercube sampling to generate a training population of $N=50$ samples and a test population of $N=25$ samples from Gaussian distribution. The data generation is performed in $A D S$ schematic simulation using the equivalent circuit model of Fig. 8(b). The statistical mapping is formulated as a linear function of (1)

$$
\begin{aligned}
& \text { Line } W_{\text {map }}^{k}=a_{1}^{k} \cdot \text { LineW }+a_{2}^{k} \cdot I D_{1} \\
& I D_{1 \text { map }}^{k}=b_{1}^{k} \cdot \text { LineW }+b_{2}^{k} \cdot I D_{1}
\end{aligned}
$$

where $\boldsymbol{\phi}^{k}=\left\{\boldsymbol{a}^{k}, \boldsymbol{b}^{k}\right\} \quad(k=1,2, \ldots, N)$ contains the mapping parameters for the $k^{\text {th }}$ statistical sample. In (9), the original explicit inputs are mapped to new inputs, with the implicit inputs being represented by the mapping parameters $\phi^{k}$.

The statistical mapping is obtained through a standard parameter extraction procedure as described in Section II.B, where mapping parameters $\boldsymbol{a}$ 's and $\boldsymbol{b}$ 's are optimized such that for each training sample, the statistically wrapped nominal model is able to produce a good fit to the data. The equivalent circuit model with the nominal input values is used as the nominal model. Figure 9 shows the probability density plot of the extracted $a_{1}$ and $b_{2}$. Similar to the first example, the extracted parameters closely follow Gaussian distribution. The means and standard deviations of the extracted parameters are

\begin{tabular}{|c|c|c|c|c|}
\hline & $a_{1}$ & $a_{2}$ & $b_{1}$ & \\
\hline & & 0.6625 & -0.1348 & 0.0105 \\
\hline & 0.6625 & 1.0000 & & \\
\hline & -0.13 & -0.23 & 1.0000 & \\
\hline & & -0.1667 & 0.3635 & 1.00 \\
\hline
\end{tabular}
shown in Table $\mathrm{V}$ with the correlation matrix as

TABLE V

EXTRACTED STATISTICS OF THE MAPPING PARAMETERS FOR THE SPIRAL INDUCTOR EXAMPLE

\begin{tabular}{|c|c|c|}
\hline \hline Mapping Parameters & Mean Values $(\boldsymbol{\mu})$ & Standard Deviation $(\boldsymbol{\sigma})$ \\
\hline$a_{1}$ & 1.0670 & 0.0338 \\
\hline$a_{2}$ & $0.1568 \mathrm{e}-10$ & $0.2120 \mathrm{e}-10$ \\
\hline$b_{1}$ & $1.5020 \mathrm{e}-14$ & $2.3078 \mathrm{e}-14$ \\
\hline$b_{2}$ & 1.0172 & 0.1023 \\
\hline \hline
\end{tabular}
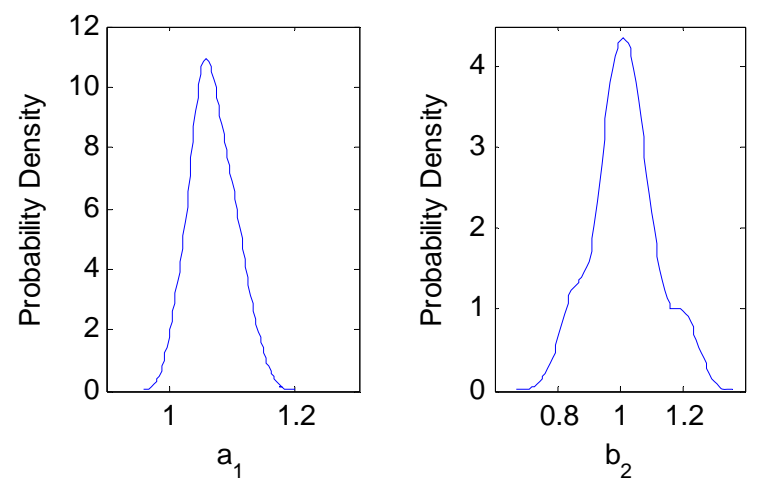

Fig. 9. Probability density plot of the extracted mapping parameters. Gaussian distribution is observed for the spiral inductor example. 
To verify the portable mapping, two different nominal models are used, represented by the fast model in the equivalent circuit (EC) form in $A D S$, and the fine EM accuracy model obtained from Momentum simulation. The mapping network with its extracted statistics in (10) and Table $\mathrm{V}$ is applied to both nominal models. Figure 10 compares the statistical means and standard deviations of inductance $L$ and quality factor $Q$ using these two different nominal models with the portable mapping, and good statistical consistency between the responses from the test population and that from the model is observed. We further studied the portability of the mapping to two new spiral inductors with different nominal shape parameters, where line width of $16 \mu \mathrm{m}$ and 25 $\mu \mathrm{m}$, line spacing of $8 \mu \mathrm{m}$ and $15 \mu \mathrm{m}$, and longitudinal and transverse inside diameters of $90 \mu \mathrm{m}$ and $200 \mu \mathrm{m}$ are used. The statistical $L$ and $Q$ for the two new structures are shown in Fig. 11, which again shows that the statistical mapping extracted from the original structure of Table IV can be carried to a new EM structure for accurate statistical prediction. In addition, reduced modeling cost by the proposed technique is also observed. For a training population of 100 samples, the overall modeling time by the proposed technique was 23.2 seconds using the fast equivalent circuit nominal model, while data generation for 100 samples using the fine EM accuracy model from Momentum simulation already required 34.88 minutes. This speedup can be very valuable for complicated EM modeling problems.

\section{CONCLUSION}

A portable mapping technique is developed for costeffective statistical modeling of passive components, as a flexible alternative to existing modeling methods. The portability of statistical mapping network enables fast model development and flexible model usage. The smart sampling method retains statistical reliability with smaller sample size, thus further reduces modeling cost. A single statistical mapping extraction can provide a set of statistical models of various accuracies for use in different design stages. This technique has simple formulation and is simulator independent. The portable statistical mapping is easy to implement and is simulation-ready.

\section{ACKNOWLEDGMENT}

The authors would like to thank Prof. Q. J. Zhang at Carleton University, Ottawa, ON, Canada, for valuable discussions and insights throughout this work.

\section{REFERENCES}

[1] M. Meehan and J. Purviance, Yield and Reliability in Microwave Circuit and System Design. Boston, MA: Artech, 1993.

[2] Z. Yu and C. C. McAndrew, "RF CMOS is more than CMOS: modeling of RF passive components," IEEE Custom Integrated Circuits Conf., San Jose, CA, Sept. 2009, pp. 407-414.

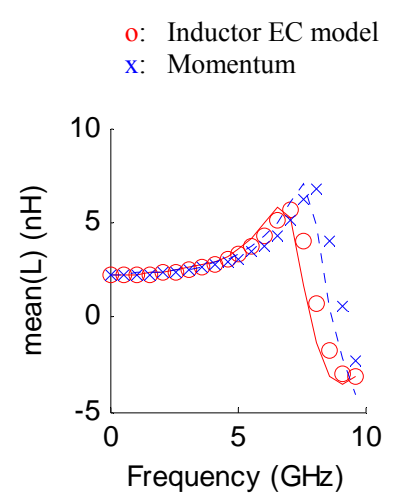

$$
\begin{aligned}
& \text { - : Mapped EC model } \\
& --- \text { : Mapped Momentum model }
\end{aligned}
$$
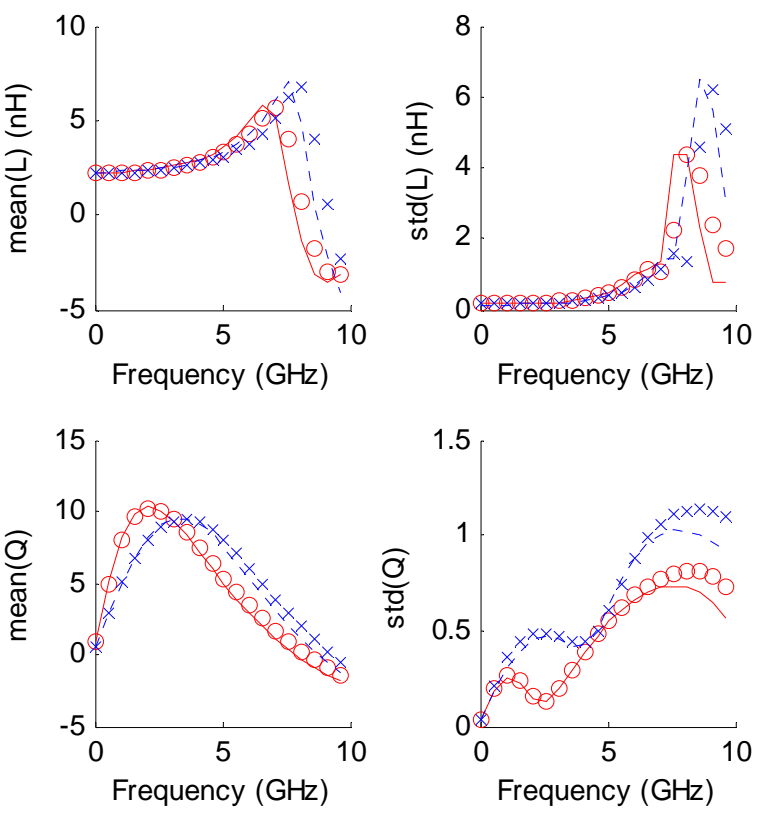

Fig. 10. Means and standard deviations of inductance $\mathrm{L}$ and quality factor $\mathrm{Q}$ of the spiral inductor example. Comparison is between the test population and the statistical mapped model. The same mapping developed from equivalent circuit (EC) nominal model is applied to the other nominal model using Momentum simulation data. The portable statistical mapping estimated the statistical behavior for both nominal models.

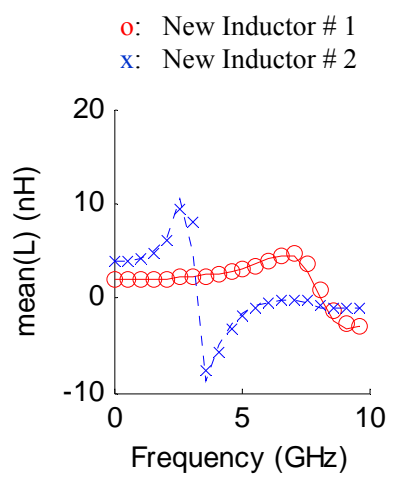

— : Mapped Model for Inductor \# 1 --- : Mapped Model for Inductor \# 2
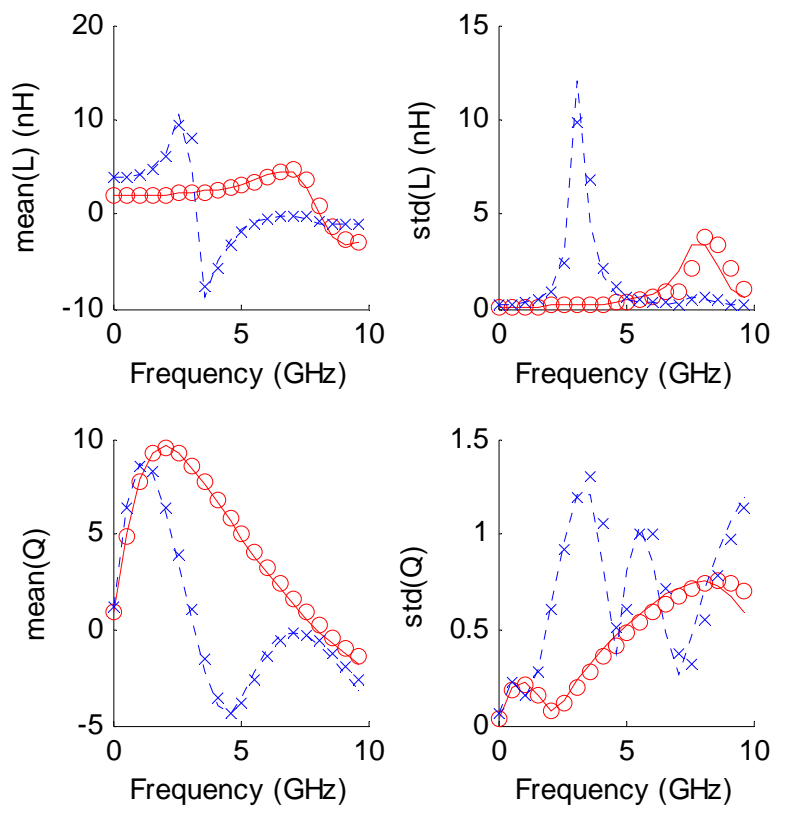

Fig. 11. Means and standard deviations of inductance $L$ and quality factor $Q$ of two new spiral inductors with different shape parameters. Comparison is between the test population and the statistical mapped model. The portable statistical mapping developed from the original spiral inductor captures the statistical behavior for both new structures. 
[3] I. Yun, R. Poddar, L. Carastro, M. Brooke, and G. S. May, "Statistical modeling of 3-D parallel-plate embedded capacitors using Monte Carlo simulation," Electronics and Telecommunications Research Institute Journal, vol. 23, no. 1, pp. 23-32, Mar. 2001.

[4] P. Cox, P. Yang, S. S. Mahant-Shetti, and P. Chatterjee, "Statistical modeling for efficient parametric yield estimation of MOS VLSI circuits," IEEE Trans. Electron Devices, vol. 32, no. 2, pp. 471-478, Feb. 1985.

[5] R. Jiang, W. Fu, J. M. Wang, V. Lin, and C. C.-P. Chen, "Efficient statistical capacitance variability modeling with orthogonal principle factor analysis," IEEE/ACM Int. Conf. on Computer-Aided Design, San Jose, CA, Nov. 2005, pp. 683-690.

[6] A. A. Mutlu and M. Rahman, "Statistical methods for the estimation of process variation effects on circuit operation," IEEE Trans. Electron Packaging Manufacturing, vol. 28, no. 4, pp. 364-375, Oct. 2005.

[7] A. V. Sathanur, R. Chakraborty, and V. Jandhyala, "A hierarchical electromagnetic-circuit technique for statistical analysis of RF circuits in the spectral domain," IEEE Trans. Microw. Theory Tech., vol. 57, no. 4 , pp. 796-806, Apr. 2009.

[8] J. W. Bandler, Q. S. Cheng, S. A. Dakroury, A. S. Mohamed, M. H. Bakr, K.Madsen, and J. Søndergaard, "Space mapping: The state of the art," IEEE Trans. Microw. Theory Tech., vol. 52, no. 1, pp. 337-361, Jan. 2004.

[9] J. E. Rayas-Sánchez and V. Gutiérrez-Ayala, "EM-based Monte Carlo analysis and yield prediction of microwave circuits using linear-input neural-output space mapping," IEEE Trans. Microw. Theory Tech., vol. 54, no. 12, pp. 4528-4537, Dec. 2006.

[10] S. Koziel, J. W. Bandler, A. S. Mohamed, and K. Madsen, "Enhanced surrogate models for statistical design exploiting space mapping technology," in IEEE MTT-S International Micro. Symp. Dig., Long Beach, CA, June 2005, pp. 1609-1612.

[11] L. Zhang, K. Bo, Q.-J. Zhang, and J. Wood, "Statistical space mapping approach for large-signal nonlinear device modeling," in 36th European Microwave Conference EuMC Symp. Dig., Manchester, UK, Sept. 2006, pp. 676-679.

[12] L. Zhang, Q.-J. Zhang, and J. Wood, "Statistical neuro-space mapping technique for large-signal modeling of nonlinear devices," IEEE Trans. Microw. Theory Tech., vol. 56, no. 11, pp. 2453-2467, Nov. 2008.

[13] W. G. Cochran, Sampling Techniques, 3rd edn. New York, NY: John Wiley and Sons, 1977.

[14] M. D. McKay, R. J. Beckman, and W. J. Conover, "A comparison of three methods for selecting values of input variables in the analysis of output from a computer code," J. Technometrics, vol. 21, no. 2, pp. 239245, May 1979.

[15] E. J. Pebesma and G. B. M. Heuvelink, "Latin hypercube sampling of Gaussian random fields," J. Technometrics, vol. 41, no. 4, pp. 303-312, Nov. 1999.

[16] R. L. Iman and W. J. Conover, "A distribution-free approach to inducing rank correlation among input variables," Comm. in Stat. - Simulation and Computation, vol. 11, no. 3, pp. 311-334, 1982.

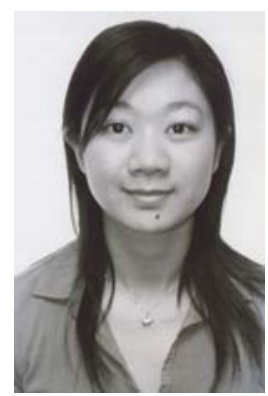

Lei Zhang (S'03-M'09) received her B.Eng. degree in electrical engineering (major) and economics (minor) from Tianjin University, Tianjin, China, in 2000, and M.A.Sc and Ph.D. degrees both in electrical engineering from Carleton University, Ottawa, ON, Canada, in 2003 and 2008, respectively.

She is currently a Modeling Engineer in the RF Modeling Team of the RF Division at Freescale Semiconductor Inc., Tempe, AZ. Her expertise includes space mapping, neural networks, automatic model generation of passive and active components and application of computer-aided design for RF circuits and systems. She has over 20 technical papers and articles published in journals, conference proceedings, and international workshop notes.

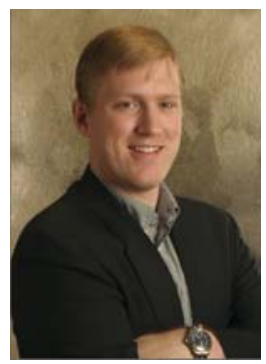

Peter H. Aaen (S'93-M'97-SM'09) received the B.A.Sc. degree in engineering science, the M.A.Sc. degree in electrical engineering both from the University of Toronto, Toronto, ON., Canada, and the $\mathrm{PhD}$ degree in electrical engineering from Arizona State University, Tempe, in 1995, 1997 and 2005 , respectively.

He currently manages the RF Modeling and Measurement Technology Team of the RF Division at Freescale Semiconductor Inc., in Tempe, AZ. He co-authored Modeling and Characterization of RF and Microwave Power FETs (Cambridge University Press, 2007) and has authored or over thirty papers, articles and workshops in the fields of electromagnetic simulation, package modeling, and microwave device modeling and characterization.

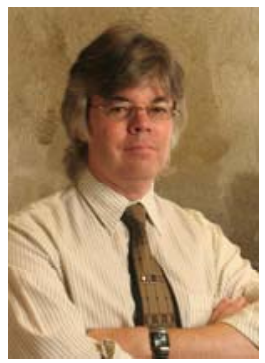

John Wood (M'87-SM'03-F'07) received B. Sc. and $\mathrm{Ph} . \mathrm{D}$. degrees in Electrical and Electronic Engineering from the University of Leeds, in 1976 and 1980, respectively.

$\mathrm{He}$ is currently Senior Principal Member of Technical Staff with Maxim Integrated Products, working on the modeling and design of envelopetracking solutions for mobile phones. He was a Distinguished Member of the Technical Staff in the RF Division of Freescale Semiconductor, where he worked from 2005--2011. His areas of expertise include the development of nonlinear compact device models and behavioral models for RF power transistors and ICs, the understanding of the impact, characterization, \& control using digital pre-distortion (DPD) of nonlinearities and memory effects in high-efficiency PAs. From 1997--2005 he worked in the Microwave Technology Center of Agilent Technologies, developing largesignal and bias-dependent linear FET models for mm-wave applications, and nonlinear behavioral models using LSNA measurements and nonlinear system identification techniques.

$\mathrm{He}$ is author or co-author of over 120 papers and articles. He is a Fellow of the IEEE, and a member of the Microwave Theory and Techniques, and Electron Devices Societies, and is a member of ARFTG Executive Committee. $\mathrm{He}$ is a Distinguished Microwave Lecturer for MTT Society. He is currently Editor-in Chief of the IEEE 'Microwave' magazine. 